\title{
Anaesthetic management of a patient with Phaeochromocytoma in a low resource setting: a case report
}

\author{
Adigun $\mathrm{TA}^{1 *}$, Ayandipo $\mathrm{OO}^{2}$ and Idowu $\mathrm{OK}^{1}$ \\ ${ }^{1}$ Department of Anaesthesia, University College Hospital, Ibadan, Nigeria \\ ${ }^{2}$ Department of Surgery, University College Hospital, Ibadan, Nigeria
}

\begin{abstract}
Background: Phaeochromocytoma is a rare catecholamine producing tumor of the adrenal gland. The anaesthetic management for the excision of this tumor can be challenging because of the haemodynamic fluctuations encountered preoperatively, during induction of anaesthesia, manipulation of the tumor and after the excision of the tumor.

Objective: To report a case of a 36-year-old Nigerian female who presented with phaeochromocytoma for surgical excision.

Methods: Combined general and epidural anaesthesia was used in the patient. Preoperatively, the patient presented with high blood pressure which was adequately controlled with alpha and beta blockers. Intraoperatively she developed severe hypertension during the manipulation of the tumor this was managed pharmacologically with labetalol, Magnesium sulphate and epidural anaesthesia while hypotension that developed after the excision of the tumor was managed with intravenous fluid and vasopressors. Postoperatively she was transferred into the intensive care unit for monitoring and pain management, she made an uncomplicated recovery.
\end{abstract}

Conclusion: It is therefore required that the anaesthetist be familiar with the physiology of the disease and pharmacology of drugs to treat the fluctuating haemodynamics to achieve a good outcome.

\section{Introduction}

Pheochromocytoma is a rare catecholamine secreting tumor that arises from the chromaffin cells of the adrenal medulla and other paraganglia tissue of the sympathetic nervous system. Adrenal is the origin for the majority of the tumor accounting for $80 \%$ and the rest are from extra adrenal sites [1].

It has an incidence of 2-8 per million populations per year in USA [2]. The actual incidence in Nigeria is however unknown apart from few reported cases due to rarity of the tumor in our environment [3-5].

Pheochromocytoma may also be a part of several endocrine abnormalities termed multiple endocrine neoplasm (MEN) syndrome type 2 consists of parathyroid hyperplasia, Pheochromocytoma and medullary thyroid carcinoma or in other familial diseases such as Von Hippel-Lindau disease and neuro fibromatosis [1].

The classic triad of headache, palpitation and diaphoresis may be seen in $40 \%$ of cases due to the effects of excessive secretion of catecholamines (norepinephrine, epinephrine or dopamine) [6]. Other signs and symptoms may be non-specific like flank pain, anxiety, pallor, visual blurring, weight loss, hyperglycemia and $50 \%$ of patients have severe hypertension refractory to conventional treatment causing prevalence of $0.1 \%$ of hypertensive population [6].

Mortality is high if patient is not well optimized before surgery due to the outpouring of catecholamine resulting in a very high blood pressure encountered preoperatievely, during laryngoscopy, intubation as well as during manipulation of the tumor. Appropriate preoperative medical management dramatically decreases perioperative morbidity and mortality in these patients.

We present the anaesthetic management of a 36-year-old female with Pheochromocytoma that underwent left adrenalectomy with a good outcome.

\section{Case Report}

A 36-year-old Nigerian female presented to the surgeon with a year history of left lumbar pain, vomiting and sweating along with hypertension. There was no history of seizure and loss of consciousness. Examination was essentially normal except for high blood pressure 170/100 $\mathrm{mmHg}$; the jugular venous pressure was not raised, and no murmur was heard. A tentative diagnosis of pheochromocytoma was made. She underwent investigations and commenced on antihypertensives; oral Prazosin $0.5 \mathrm{mg}$ twice daily and oral Metoprolol $50 \mathrm{mg}$ once daily. Her blood pressure was controlled on these medications with blood pressure in the range of $130 / 80$ $120 / 70 \mathrm{mmHg}$.

*Correspondence to: Tinuola Abiodun Adigun, Department of Anaesthesia, University College Hospital, Ibadan, Nigeria, Tel: 2349025058328; E-mail: tonitomi2005@yahoo.co.uk

Key words: Combined general and epidural anaesthesia, phaeochromocytoma

Received: March 16, 2018; Accepted: March 27, 2018; Published: March 30, 2018 
Results of the investigations revealed a packed cell volume of $30 \%$, fasting blood sugar was $102 \mathrm{mg} / \mathrm{dl}$, the 24 hour Urinary VMA (Vanyl Mandelic Acid) concentration was $9 \mathrm{mg} \%$ (normal $<8 \mathrm{mg} \%$ ), Serum Calcium, Serum Electrolytes and urea, Thyroid function test, clotting profile and Chest X ray were all normal. Electrocardiogram showed sinus rhythm. IVU showed normal excreting kidneys. The result of magnetic resonance imaging studies showed a large, well demarcated retroperitoneal adrenal mass in the left upper abdomen localized between the aorta and vena cava, measuring approximately $9 \times 6 \times 4.5$ $\mathrm{cm}$ (Figure 1).

Patient was planned for excision of the adrenal mass and therefore referred to the anesthetist for evaluation, during the preoperative visit; she was noticed to be calm, not pale, anicteric and acyanosed. Pulse rate was 66/minute, regular and good volume. Blood pressure recorded over the right upper limb was 130/85 mm Hg in supine and 125/75 mm $\mathrm{Hg}$ in standing position. Systemic examination and airway assessment were essentially normal. She was assigned ASA [3]. High risk informed consent was taken, and she continued with her antihypertensives medications till the morning of surgery and diazepam $10 \mathrm{mg}$ at night and morning of surgery and was fasted overnight. An intravenous saline solution $1000 \mathrm{ml}$ was allowed to run 8 hours over the night. She was scheduled for left suprarenal mass removal with or without a left nephrectomy.

\section{Anesthetic management}

On arrival in the theatre, an 18-gauge intravenous cannula was inserted, and lactated Ringer's solution was commenced. Non-invasive monitors were attached for monitoring: automated non-invasive blood pressure, pulse oximeter, electrocardiogram and temperature probe in addition to 20- gauge cannula over the radial artery for invasive blood pressure monitoring. Baseline vital signs were pulse rate 98 beats per minute, blood pressure was $140 / 90 \mathrm{mmHg}$ and oxygen saturation was $98 \%$.

In the sitting position, epidural needle was inserted at $\mathrm{L} 1 / 2$ under aseptic technique and epidural catheter was inserted after a test dose of $3 \mathrm{mls}$ of lidocaine without adrenaline. Epidural analgesia was activated with $10 \mathrm{ml}$ of $0.25 \%$ plain bupivacaine after a test dose.

Patient was pre-oxygenated with $100 \%$ oxygen, she was then premedicated with fentanyl 100 ug and induced with propofol $150 \mathrm{mg}$. Muscle paralysis was with vecuronium $6 \mathrm{mg}$, the endotracheal intubation was with $7.00 \mathrm{~mm}$ size endotracheal tube and nasogastric tube was put in place, the patient mechanically ventilated with a tidal volume of 8 $\mathrm{ml} \mathrm{kg}{ }^{-1}$ at a rate of 14 breaths $\mathrm{min}^{-1}$. The patient's end-tidal Pco2was between $35-40 \mathrm{~mm} \mathrm{Hg}$. Anaesthesia was maintained with isoflurane $1.2 \%$ MAC in $100 \%$ of oxygen. Patient was essentially stable until during the tumor manipulation. She became haemodynamic unstable, blood pressure shot up to $190 / 105 \mathrm{mmHg}$ with increased heart rate up to135 beats/minute. This was managed with IV boluses of labetalol 5 $\mathrm{mg}$, IV magnesium sulphate 2-gram stat, topping up the epidural 10 $\mathrm{mls}$ of $0.5 \%$ of plain Bupivaine and increasing the depth of inhalational anaesthetic agent. The adrenal gland was morbidly adherent to the left kidney, so she had left nephrectomy with adrenalectomy (Figure 2).

After tumor excision, the patient developed profound hypotension. This was corrected with volume replacement, 1.5 litres of Ringers lactate and vasopressors ephedrine $3 \mathrm{mg}$ boluses and continuous infusion of Dopamine at $5 \mathrm{ug} / \mathrm{kg}$. The surgery lasted 2 hours and estimated blood loss was about $500 \mathrm{ml}$ for which a unit of fresh whole blood was transfused. Extubation of endotracheal tube was done at the end of the surgery on the table after reversal of the residual muscle paralysis with IV $0.4 \mathrm{mg}$ of glycopyrrolate and IV $2.5 \mathrm{mg}$ neostigmine.

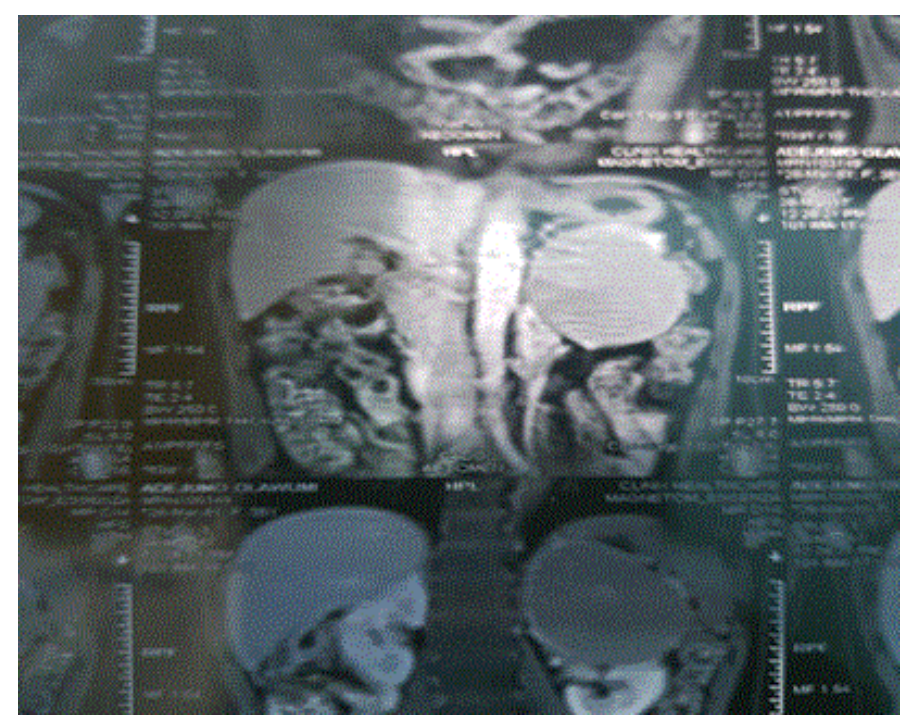

Figure 1. Magnetic Resonance Imaging of the abdomen.

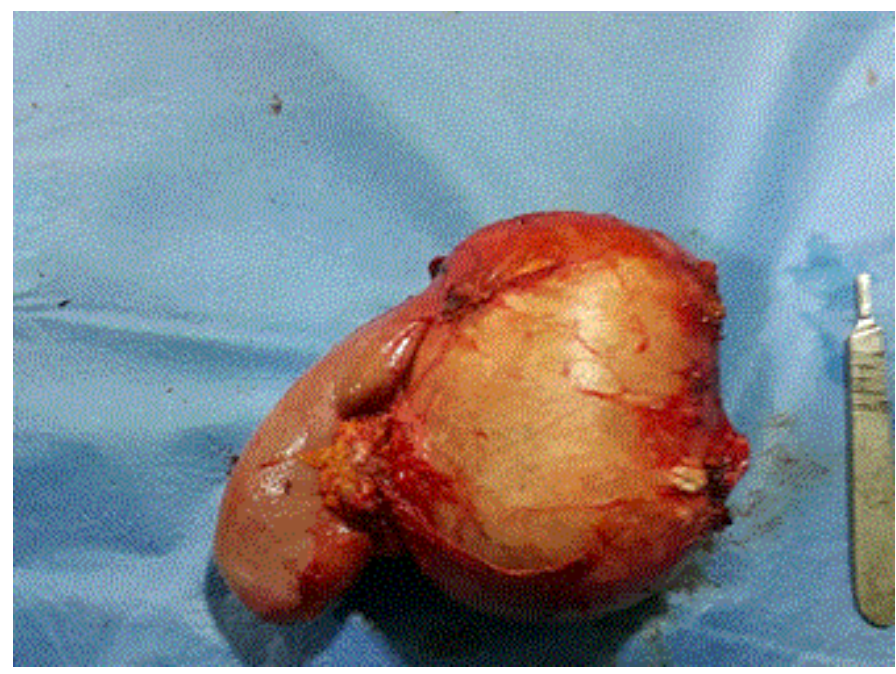

Figure 2. Intraoperative image of kidney and the supra renal mass.

She was transferred to the Intensive Care Unit (ICU) for monitoring. Post-operative analgesia was via the epidural catheter for the first 24 hours afterwards $4 \mathrm{mg}$ of intravenous morphine 4 hourly. Her postoperative course was essentially uneventful and was discharged from ICU on the third day and from the hospital on the seventh day, with no complications, signs or symptoms of hypertension on follow-up 2 months after surgery.

\section{Discussion}

Phaeochromocytoma is a rare neuroendocrine tumor that originates from the chromaffin cells of the adrenal medulla and other paraganglia tissue of the sympathetic nervous system. Adrenal is the origin for the majority of the tumor in $80 \%$ of cases and the rest are from extra adrenal sites $[1,2]$.

The combination of regional and general anaesthesia provides satisfactory condition for excision of the tumor in our patient. Previous successful use of combined epidural and general anaesthesia as anesthetic technique for the surgical excision of pheochromocytoma was documented by Sotunmbi et al as a case report in our institution3. Several anaesthetic options for excision of phaeochromocytoma 
have been reported, these include general anaesthesia only, regional anaesthesia only or the combination of both $[7,8]$. The choice of anaesthesia only does not influence the outcome of surgery but the sound knowledge of pharmacology of the drugs used [9]. It has been reported that $25-50 \%$ of patients die during induction of anaesthesia so careful preoperative control of blood pressure is essential and surgery should only be done as elective after good blood pressure control [9].

Roizen et al. proposed a set of criteria to effectively measure the adequacy of preoperative optimization prior to excision of phaeochromocytoma, a) absence of in-hospital blood pressure of greater than $160 / 90 \mathrm{mmHg} 24$ hours prior to surgery, b) absence of orthostatic hypotension less than $80 / 45 \mathrm{mmHg}, \mathrm{c}$ ) absence of ST or T wave changes one week prior to surgery and d) absence of more than five premature ventricular contraction per minute [10]. In our case report, the patient met the Roizen criteria.

Preoperative blood pressure control using alpha and betaadrenergic blockade has been the main stay of preoperative preparation [11]. In our case report, Prazosin was used; it has the advantages of short elimination half-life of 2 to 3 hours, less reflex tachycardia, less pre and postoperative hypotension although phenoxybenzamine, an old drug is most frequently used, it is a long acting, non- selective alpha blocker; the drug is usually started in a small dose and increased until signs and symptoms of postural hypotension, dizziness and tachycardia occurred. These effects can persist into the postoperative period hence the drug is usually stopped 48 hours before surgery [8].

Beta adrenergic blocker, metoprolol was used preoperatively after alpha blocker for optima blood pressure control in our study. Beta blocker is used to limit the signs and symptoms of tachyarrythmias; it should therefore be avoided in patients with catecholamine induced cardiomyopathy as it can lead to intractable hypotension and bradycardia [12]. Other beta blockers like propanolol, bisoprolol or atenolol have also been used with good results to control blood pressure in some cases $[8,11]$. Alpha and beta blocker combination such as labetalol has been used for pre and intraoperative blood pressure control with good result [13]. This patient had labetalol for intraoperative blood pressure control only. Calcium blocker such as Nifedipine or Nicardipine has also been used to control blood pressure in the preoperative period although calcium blocker was not used at all in our patient.

The patient was premedicated with diazepam and drugs like sodium thiopentone, morphine, mivacuronium and atracurium which potentially release histamine because of the risk of provoking catecholamine release from chromaffin granules were avoided in the patient. Although Prys-Roberts et al. used Atracurium without untoward side effects in the management of phaeochromocytoma [8]. Intraoperative hypertensive crisis was pharmacologically managed in this case with bolus doses of labetalol and magnesium sulphate and epidural anaesthesia. Magnesium sulphate is a calcium antagonist and also inhibits the release of catecholamine. It can be considered as an attractive option for catecholamine blockade in patients undergoing pheochromocytoma excision [14]. James and colleagues pioneered the use of magnesium sulphate (bolus and infusion) to control intraoperative hypertension in a case series of 17 patients, good stability of intraoperative blood pressure was achieved in 12 out of 17 patients. Magnesium sulphate was used successfully with epidural anaesthesia in another study [15]. Epidural anaesthesia has the advantages of intraoperative blood pressure control and postoperative analgesia.

After the removal of the tumor, hypotension which may be refractory to volume replacement may occur due residual effect of preoperative alpha-adrenergic blocker or haemorrhage. Our patient responded to boluses of ephedrine and dopamine infusion. Although epinephrine, nor-epinephrine and phenylephrine were used in some cases with good report [13].

Monitoring is an important part of management in this patient, invasive blood pressure monitoring is the gold standard in the pheochromocytoma resection for beat-to-beat blood pressure monitoring allowing for close haemodynamic monitoring in addition to other non- invasive monitoring that was used in our patient. Central venous pressure (CVP) monitoring is essential for more accurate fluid management and pulmonary arterial wedge catheter (PAWP) for patients with poor left ventricular function from a catecholamine cardiomyopathy, pulmonary hypertension, or significant myocardial disease [13]. Although CVP and PAWP were not used in our patient due to cost however their use may be more useful particularly in the unstable cardiac patient.

\section{Conclusion}

Phaeochromocytoma is rare tumor and its management is challenging to the anaesthetist but with thorough knowledge of the physiology and pharmacology of drugs good outcome is achieved.

\section{References}

1. Lender JW, Eisenhofer G, Mannelli M, Pacak K. Phaeochromocytoma. Lancet 2005; 366: $665-75$

2. Golden SH, Robinson KA, Saldanha I, Anton B, Ladenson PW (2009) Clinical review: Prevalence and incidence of endocrine and metabolic disorders in the United States: a comprehensive review. J Clin Endocrinol Metab 94: 1853-1878. [Crossref]

3. Sotunmbi PT, Shittu OB, Windokun A, Eyelade AO (2000) Combined general and epidural anaesthesia for excision of phaeochromocytoma - a unique and safe technique. Afr J Med and Med Sci 29: 319-322.

4. Adenekan AT, Faponle AF, Badmus TA, Sanusi A (2011) Anaesthetic management of a giant pheochromocytoma in a patient with a chronic renal disease. $J$ West Afr Coll Surg 1: 112-122.

5. Afuwape OO, Ladipo JK, Ogun OO, Adeleye J, Irabor ID (2009) Phaeochromocytoma in an accessory adrenal gland: Case report. Cases J 2: 6271 .

6. Bravo EL1 (1991) Pheochromocytoma: new concepts and future trends. Kidney Int 40 544-556. [Crossref]

7. Roizen MF, Horrigan RW, Koike M, Eger IE, Mulroy MF, et al. (1982) A prospective randomized trial of four anesthetic techniques for resection of pheochromocytoma. Anesthesiology 57: A43.

8. Prys-Roberts C (2000) Phaeochromocytoma: recent progress in its management. $\mathrm{Br} J$ Anaesth 85: 44-57. [Crossref]

9. Singh G, Kann P (1998) An overview of anaesthetic issues in pheochromocytoma. Ann Acad Med Singapore 27: 3-8

10. Roizen MF, Schneider BD, Hassan SZ (1987) Anaesthesia for patients with pheochromocytoma. Anaesthesiolo Clin North Am 5: 269-275.

11. Mazza A, Armigliato M, Marzola MC, Schiavon L, Montemurro D, et al. (2014) Anti-hypertensive treatment in pheochromocytoma and paraganglioma: Current management and therapeutic features. Endocrine 45: 469-478.

12. Kassim TA, Clarke DD, Mai VQ, Clyde PW (2008) Catecholamine-induced cardiomyopathy. Endocr Pract 14: 1137-1149.

13. Aliya H (2005) Perioperative management of pheochromocytoma: Anaesthetic implication. J Pak Med Assoc 57: 140-146.

14. James MF (1989) Use of magnesium sulphate in the anaesthetic management of phaeochromocytoma: a review of 17 anaesthetics. Br J Anaesth 62: 616-623. [Crossref]

15. Pivalizza EG (1995) Magnesium sulphate and epidural anesthesia in pheochromocytoma and severe coronary artery disease. Anaesth Analg 81: 414-416.

Copyright: (C2018 Adigun TA. This is an open-access article distributed under the terms of the Creative Commons Attribution License, which permits unrestricted use, distribution, and reproduction in any medium, provided the original author and source are credited. 